\title{
Gas and Solvent for dispersion for Injection/Infusion Dosage Form
}

National Cancer Institute

\section{Source}

National Cancer Institute. Gas and Solvent for dispersion for Injection/Infusion Dosage

Form. NCl Thesaurus. Code C149524.

Sterile gas and sterile solvent, either in the same compartment or in different compartments, intended for the preparation of a dispersion for injection/infusion by dispersing the gas in the solvent. 